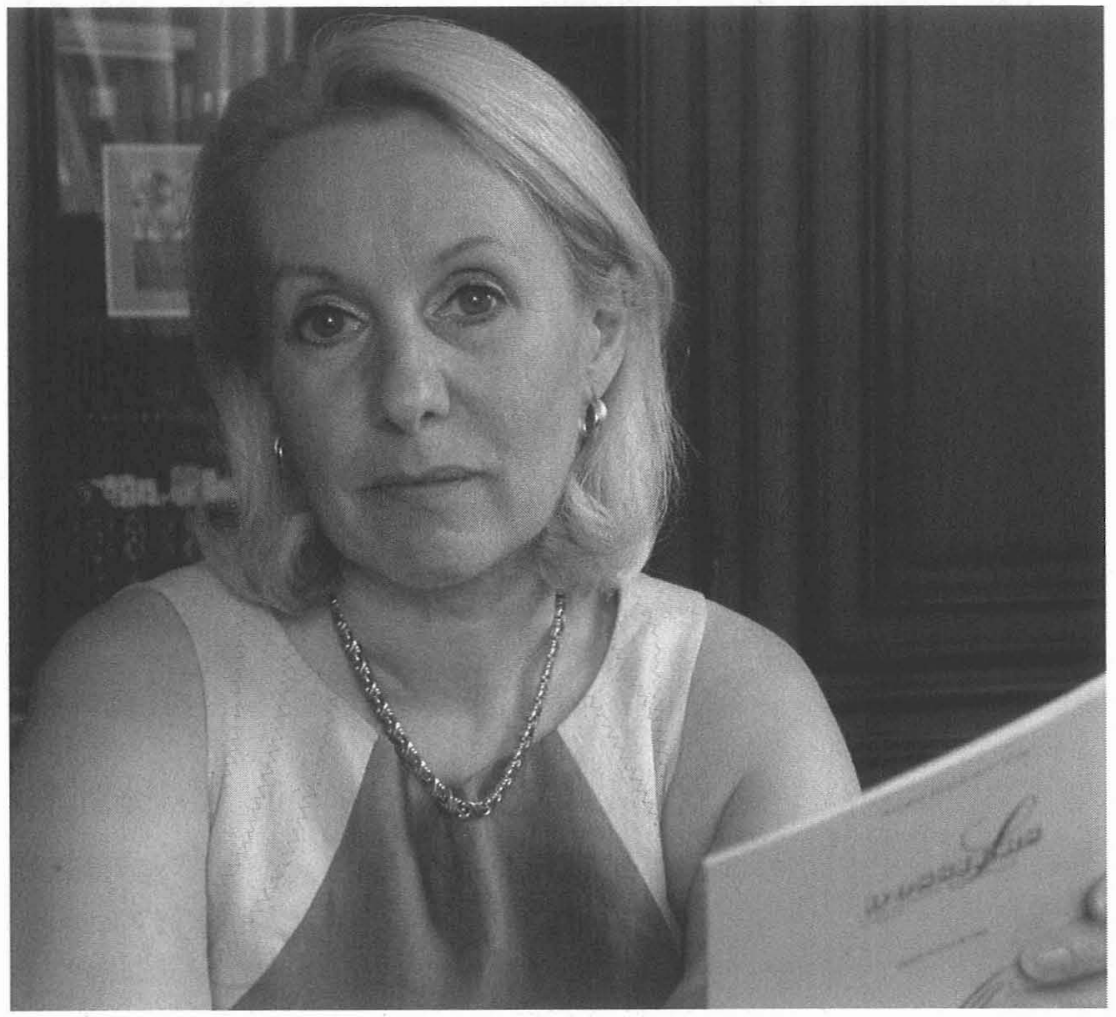

BÉATRICE LIBERT

34 - LittéRéalité 


\title{
Béatrice Libert ou les mots du corps
}

\author{
Chantal Danjou \\ Camps La Source, France
}

"Tu marches

et c'est ton pas qui donne

sens à la route

Tu marches

et c'est la route qui prend

racine en toi

en ton désir d'aller

de ce que tu fus

à ce que tu es

Tu marches

et c'est le pays traversé

qui met des ailes

à ton identité»

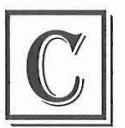

'est par ce poème que Béatrice Libert ${ }^{1}$ souhaitait la Bonne Année 2002 à ses amis, ajoutant : "que l'an neuf vous donne des ailes pour porter la lumière toujours plus loin, toujours plus haut! " Et cette marche vers la lumière - lumière intérieure, s'entend - est bien la quête identitaire d'un poète, connaissance/reconnaissance, sens/rupture de sens, lien/dislocation. Avancée initiatique mais aussi sensitive, pas alerte, buste

1 Béatrice Libert vit et enseigne à Liège.

Elle a publié plusieurs livres de poèmes, des essais ainsi que des nouvelles en revues. Critique de poésie, animatrice en ateliers d'écriture, elle écrit aussi pour la jeunesse. Passionnée par l'art sous toutes ses formes, elle collabore avec des artistes peintres, graveurs, photographes et musiciens. 
légèrement penché en avant. La route naît du rythme même de la marche, du poids de ce corps, de l'empreinte qu'il laisse, de l'exercice régulier, peutêtre quotidien, de cette marche que double la rêverie. Et de la poche de cette marcheuse infatigable qu'est Béatrice Libert tombent les mots comme autant de petits cailloux qui, chaque jour, lui rappellent l'itinéraire de la veille et l'encouragent à poursuivre la route. "L'aube surgit parfois d'un jet de cailloux ", écrit-elle dans Être au monde. J'y vois une métaphore de l'écriture, un travail, un jeu - mot voisin de jet-de construction, peut-être de hasard, avec les cailloux qui apparaissent comme des signes primaires d'écriture.

Pour le poète, la parole est fondatrice, à l'origine de toutes choses. Elle met en place le monde. Sans doute Béatrice Libert appartient-elle avant tout à une terre de langue. Sensuelle, féconde, langue du désir; dans la suite érotique Volà main nue, elle note: "Tu parles en elle / langue contre langue." À cet égard la plaquette intitulée Lalangue (écrit en un seul mot) du désir et du désarroi est essentielle dans la maturation de son projet poétique. Rien qu'un bref extrait de cette plaquette peut nous en donner la tonalité : "Suis de neige / comme on oublie le feu / un long jour de lessive / dans les grands baquets bleus". Notons l'apparente contradiction des termes de neige et de $f e u$, de désir et de désarroi, avec l'allitération surprenante. D'autre part, le lalangue en un seul mot m'a interpellée. J'y ai vu à la fois le lalein grec parler - et lallation, c'est-à-dire l'émission de sons plus ou moins articulés par l'enfant avant l'acquisition du langage. Outre le désir, je m'arrêterai à ce désarroi que j’interprète comme la difficulté de la mise en ordre des mots. Je me permets de reprendre ici la dédicace si judicieuse que Béatrice Libert m'avait faite : "cette lalangue qui est notre marais poétique originel". Par cette métaphore du marais elle souligne la notion de culture l'exploitation maraîchère du marais - d'ouverture aussi aux éléments neufs - le bassin que peut être aussi le marais, creusé à proximité des côtes pour en extraire le sel. Autant d'interprétations s'appliquant bien sûr au langage. Pour Lacan qu'elle cite, c'est par la parole que l'être humain accède à la dialectique de l'intersubjectivité, régie par le désir de l'Autre. Ainsi, cet aveu inquiétant dans Etre au monde : "Comme si je ne pouvais plus / ni parler ni écrire / ni aucun geste qui me relie aux autres / Comme si je n'avais

Dernières publications : Petit bréviaire amoureux, poèmes, Les Ecrits des Forges, Trois-Rivières; Etre au monde, poèmes, La Différence, Paris. À paraittre aux éditions Autres Temps à Marseille : Le passant fabuleux, poèmes consacrés aux ouvres de Picasso. 
plus d'espace / où me mouvoir / où mïnventer / pas même cet enclos de la cellule primitive ».

Face à ce "désarroi ", à ce "bonheur inconsolé" - titre d'un autre recueil - dans la $4^{\mathrm{e}}$ de couverture de "L'Heure Blanche ", Jean Joubert reprenait le thème essentiel du travail de Béatrice Libert, à savoir « la jubilation presque païenne " qu'éveillent en elle "les humbles présences de la vie quotidienne". Vision on ne peut plus juste de cet enracinement dans la terre, dans le silence, devant la rose trémière, voulu par le poète belge. "Chaque matin, je nais au même rituel / avec le sansonnet, le chat, l'abeille, le scarabée / Grande carnassière de l'immédiat, / jatteins le coeur d'aimer, ses rives, son delta. / Nous sommes de la tribu des longs vivants, / nous donnant sans compter à l'instant bleu ».

Alors, qui est vraiment Béatrice Libert? Comblée par le chant, le miel, l'instant? Frustrée, isolée, inquiète? Comme j'aime à traquer "sous " le poète l'être, l'être humble, l'être nu ou plus exactement mis à nu et qu'à mon avis seuls de tels dénudation/dénuement permettent une authentique poursuite de l'écriture, j'ai glané dans l'une de ses lettres quelques mots célébrant un réel pris sur le vif: "il faut saccrocher aux choses quotidiennes, acheter des fleurs et composer de beaux montages floraux : c'est bon pour le moral. »

Et pourtant, à la même époque, qu'avouait-elle en première page de "L'Heure Blanche "? "Mes pas me portent vers moi-même / et vers le centre de l'univers / que je n'atteins jamais / par manque de dénuement / de silence ou d'ivresse "

Ce que j'aimerais proposer? L'exercice périlleux d'une lecture de ses tout-derniers recueils à la lumière de ce poème courageux. Où vont les pas du poète, où la portent-ils dans Être au monde, dans Petit bréviaire amoureux? A-t-elle consenti à une nudité authentique? A-t-elle assez abandonné? C'est ce qu'elle interroge - me semble-t-il - ce qu'elle demande à son lecteur aussi. Le fait que ces deux recueils - Etre au monde et Petit bréviaire amoureux - tout en accueillant des inédits, reprennent des recueils antérieurs ne relève pas d'une démarche anodine ni facile. Volonté d'une rétrospective, d'un bilan, avant d'aborder un nouveau virage dans sa route de poète? Sans doute. Peut-être aussi l'espoir de rassembler dans une sorte d'unité de temps et de lieu ces fragments d'identité qui constituent tout être.

"Les mots dessinent le corps / Les lèvres en font le tour ". Étrangement ou pas, c'est l'image du tour du potier qui me vient à l'esprit. Dans l'exercice amoureux comme dans la pratique poétique, l'un l'autre s'influençant, il y a les notions de modelage, d'élagage, de dénudation qui donnent à voir les 
formes en train de naître, les épousailles qui se constituent, sorte d'invention consentie à l'amour, à l'écriture. Se posant les lèvres suggèrent le corps, le font vibrer, le nomment et le disant le font advenir. Plusieurs textes reprennent l'image du travail du potier. "Dans nos paumes d'argile bien à l'abri de notre unicité ", écrit-elle dans le poème intitulé " Exil ». Effet Pygmalion encore dans un autre texte - «Fontaine - «enveloppée de nuit et de salive / Fontaine femelle sculptée par l'amant». Ce n'est sans doute pas par hasard que chacun de ces vers clôture le poème, renforçant ainsi l'impression sur le lecteur.

Il s'agit, me semble-t-il, d'un travail qui privilégie les attitudes, sorte d'instantané sur une silhouette, sur une ombre. Les verbes utilisés sont pour la plupart des verbes de peu de mouvement. Ainsi : "Debout à la fenêtre / légèrement fardée / par le jour naissant / elle se penche vers toi ». Le regard de Béatrice Libert est un regard qui surprend, qui dérobe, qui caresse, presque un regard de voyeuse. "Elle vient voler ton âme / enfiler le manteau de tes fantasmes / courir menue sur le clavier de ta déroute. "

La nudité est un travail dans l'immobile, initiant un voyage intérieur, une descente dans l'imaginaire, presque dans le hors-vie, consentant à la petite mort. Ainsi peut-elle écrire : "Tu as surgi de l'immobile ", prolongé par "Tu rêves dans l'outre-corps", "Voyager en nous pacifie nos doutes". Travail dans l'immobile, ai-je dit, je pourrais ajouter dans la veille - une veille patiente - et la lenteur. "Il reste un pan d'éternité / à pétrir en nos mains. "L'éternité, ou du moins le sentiment que l'on en a, naît d'actes longs, patients, inscrits dans la durée et la chaleur. L'éternité prend dans une matière, sensuelle, caressante, s'entretient dans un monde clos " moisson/fenil/mains " - presque un huis clos. L'impression de lenteur "laisser infuser/dormir/rester" - est telle que ce sont les verbes, les choses nommées, renouant en cela avec la tradition biblique du verbe fait chair, qui font advenir, qui donnent trace et mesure, qui délient et suspendent le temps. J'ai envie de dire que chez Béatrice Libert, tout est dans la sensation car c'est de ce qui est touché, palpé, modelé puis articulé, proféré que s'élabore le poème. C'est peut-être pour cette raison que les mots qu'elle choisit juxtaposent souvent des extrêmes dans la sensation; à titre d'exemple, je citerais "sourciers/brasiers " ou " acierlamande " ou encore "source/rocher ", "obscurllumière »... Cette palette variée des sensations est là pour signifier que tout s'effectue dans la nuance, jusqu'à la plus imperceptible nuance, jusqu'au moindre souffle. L'éternité qu' elle entrevoit ne fige pas; elle poursuit ce qui est commencé comme le songe, chez l'homme apparemment endormi, révèle, éclaire, apprend le sens caché des choses. Elle inscrit l'histoire dans les lignes, les rides et les entailles du 
corps. "Nous voici vieux d'un long matin sans âge / qui court à la fontaine bien plus vite que nous / Et notre pas s'alentit et nous rentrons en nous / à tâtons solitaires désorientés ». De quelle fragilité aussi est faite cette éternité! L'être est plutôt pressenti que vécu, comme s'il était en suspens : " mais un pollen sur nos ailes invisibles appelle à la douceur. "Il serait plus juste de parler, comme le poète le fait judicieusement, d' "éternité provisoire ».

Il y a toujours un mouvement qui va de l'intime à l'universel, de l'instant à l'éternité. Un travail sur le rythme le favorise. D'abord, dans la répétition de certains mots qui scandent le poème. C'est le cas de "matin", repris parfois par "aube " dans le texte qui initie Etre au monde. Ainsi lisons-nous successivement : "Matin qui nous rapproche de toutes les aurores - matin léger! - Aube en nous - matin ouvert - Matin sous le vert - des semeurs de matins ». Mis en exergue, rehaussé par une expansion du nom relative, adjectif qualificatif ou complément du nom - ce " matin du monde " est comme déifié. "Il tombe un lait de silence et de fruit. Seule, je suis l'Adam et L'Ève de tous les petits matins à cueillir dans nos paumes. »

Et quand Béatrice Libert écrit : "En sa paume / le vent de l'histoire / tient les hommes debout ", ne serait-ce pas aussi suggérer un temps à l'intérieur même du temps, moment amoureux, instant de la création, et les amants, symboliques, n'étant là que pour extraire le présent de deux entités perdues dans le passé? "J'ai mis les mots qu'il fallait dans ma bouche et je suis venue vers toi m'en régaler à tes genoux Ils échappent à l'incandescence de l'acte conjugué à tous les imparfaits les passés simples et les impératifs [...] Ah! Sache mon doux ami que jengrange une fournaise de vocables pour quand je viendrai l'hiver nue coulante et remuée avec les mots quill faut juste à point dans ma bouche."

Dans l'Avant-Dire, initiant le Petit bréviaire amoureux, Marcel Moreau note: "Non seulement ils se font bien l'amour avec les corps, mais ils le font bien aussi avec les mots du corps ", poursuivant par : "Il y a un corps verbal dans ce corps charnel. "Oui, sensations et mots sont étroitement liés, renforçant la présence du corps. Des expressions étonnantes telles «tu la touches de la voix ". Comme le souligne Marcel Moreau : "Dans un corps follement ou éperdument épris, donc orgiaque, tout fait l'amour avec tout: l'odorat avec l'oü̈e, le goût avec la vue, le toucher avec la brûlure, le sang avec la salive, l'abysse avec l'épiderme, la déraison avec les sentiments et même les impuretés avec la frầcheur."

Le corps est habité par la voix, résonance de cette voix, de ces voix qui se répondent ou se mêlent. Le corps est chant ou cri. Une expression surprenante est employée à cet égard : "polyphonie luxurieuse ». Parfois le corps entre dans le silence. Les mots se juxtaposent, le texte se désarticule, 
se désaccorde comme le corps évoqué, sans lien, sans coordination, sans articulation entre ses différentes parties : "la main la nuque / le ventre le nombril ». Des associations, enfin, permettant un travail sonore signifiant comme avec "le sens du sang », cours - le cours d'un fleuve - déplacement, signification, justification, tout peut être compris dans ce seul mot de sens. Béatrice Libert affectionne d'ailleurs ces termes à double registre de concret et d'abstrait. En témoigne cet autre passage : "Elle a choisi la nudité / sous l'éloquence d'un long déshabillé ", provocation contenue dans cette éloquence, éveil du désir, mais aussi mot, discours de célébration.

"Sous son écorce vibre / l'aiguille secrète du désir ", corps charnel et corps textuel, l'un par l'autre s'accomplissant. Écorce, peau, page, aubier, racine, feuillage, une telle diversité de matière intensifie l'importance de l'élagage et de la dénudation. Nue, la femme l'est intimement et spirituellement, en grande complicité avec les éléments, d'une sensualité animale et heureuse, même de façon fugace. Et la voilà, vêtue/dévêtue, voilée/dévoilée par le jour, l'iris, le miroir, l'aube, le ciel, l'arbre. "Je peux paraître nue / tu me dévêts encore "; "Elle ôta sa robe / puis une autre / et une autre [...] jusqu'à la peau / cette autre robe ". La nudité doit être extrême, presque sauvage, ôtée, arrachée, écorce après étoffe, peau, végétale, animale, humaine; la nudité intègre toutes les matières sensuelles, même autres que la chair. "Regarde / Je suis plus nue quà l'origine / Tu vois en moi plus loin que l'horizon des mots / tendus entre nos chants silencieux". Un aveu poursuivi quelques textes plus loin par: "Déshabillée...ne plus penser dit-elle ". À la limite, dans ce recueil "d'encre et d'écorce »-pour emprunter l'un des titres - les pages que l'on tourne sont autant de robes ôtées...

Alors oui, revient l'aveu de cette impossibilité à être soi, complètement, intimement, et que double une quête ardente de vérité. Que représenterait la nudité ultime? Ne serait-elle pas conjointe de la séparation, de l'abandon, de la mort? Du silence aussi? "Repousses-en les limites / Entre en moi de mille façons / Que je sois alors tous les chemins du partage / toutes les rives du vouloir / toutes les portes du mystère "

Une symbolique importante dans ce travail : celle de la clef. Je l'associerais volontiers à toute une thématique autour du labyrinthe, de la noyade, de la déroute, des fantasmes. Béatrice Libert juxtapose des termes tels que "Se perdre / réinventer". N'existerait-il d'amour que d'amour dit? "Ta langue est une clef qui sait tourner / sept fois / les rêves dans la bouche." Le poème entre parfois dans l'univers du conte avec les mots traditionnels du conte qui parent le corps, enrichissent le décor, donnent au mot une tonalité magique. "Ton regard est un palais / où dansent ses envies "; "Ève / Palais savoureux "; "Elle vit / dans l'iris que tu respires / dans le muscat que 
tu croques / dans le château dont tu n'as pas la clef". Le symbolisme de la clef est sans aucun doute en relation avec son double rôle d'ouverture et de fermeture. Le pouvoir des clefs est celui qui permet de lier et de délier, de coaguler et de dissoudre, dit-on aussi. Les clefs de Janus ouvrent les portes solsticiales, c'est-à-dire l'accès aux phases ascendantes et descendantes du cycle annuel, aux temps diurnes et nocturnes. Effectivement la lumière et son double d'ombre sont très présents dans ce travail et c'est de leur achoppement que la page blanche se trouve fécondée. La couleur blanche, fréquente, presque rythmique - l'heure blanche, titre de recueil - évoquent sans doute un hors-temps, un espace aussi de recomposition, un creux, un doute, une fraude, pour reprendre des termes qui sont ceux du poète, et c'est dans cet interstice que se trame la vie, que s'élabore "le lent alphabet", que "L'Ève éclôt".

"La femme se tient seule / au milieu du milieu »; ce milieu du milieu qui m'interpelle, pourrait sans doute correspondre à ce centre de l'univers jamais atteint que je citais en début d'analyse. Au fil des expériences d'écriture et de vie, y a -t-il eu assez d'ivresse, assez de silence à travers les mots? " $U n$ bruit injecte son venin dans le silence / Guérissez-nous, feuillages où s'éponge l'azur, I troncs abrupts comme des rocs! / Qu'infuse en nous votre joie, / votre regard de renard étourdi, / de tournesols avides, de minute papillon... / Nous sommes anesthésiés. / Chaque seconde a son prix : / mille mûres dans l'interdit." 